\title{
Aerobic Exercise Alleviates Ischemia-Induced Memory Impairment by Enhancing Cell Proliferation and Suppressing Neuronal Apoptosis in Hippocampus
}

\author{
Tae-Beom Seo ${ }^{1}$, Tae-Woon $\mathrm{Kim}^{2}$, Mal-Soon Shin², Eun-Sang Ji², Han-Sam Cho², Jae-Min Lee'2, Tae-Wook Kim ${ }^{3}$, Chang-Ju Kim² \\ ${ }^{1}$ Division of Sports Science \& Engineering, Department of Biochemistry, Korea Institute of Sports Science, Seoul, Korea \\ ${ }^{2}$ Department of Physiology, Kyung Hee University School of Medicine, Seoul, Korea \\ ${ }^{3}$ Department of Physical Education, College of Physical Education, Hanyang University, Seoul, Korea
}

Purpose: Neurogenic lower urinary tract dysfunction (NLUTD) is a possible consequence of several neurological disorders. NLUTD may produce debilitating symptoms and serious complications, such as chronic renal failure, and recurrent urinary tract infections. Many animal studies of NLUTD symptoms have focused on animal models of cerebral ischemia. In the present study, we investigated the effects of treadmill exercise on memory function and its relation to cell proliferation and apoptosis in the hippocampus, following transient global ischemia in gerbils.

Methods: To induce transient global ischemia in gerbil, both common carotid arteries were occluded for 5 minutes. Gerbils in the exercise groups were forced to run on a treadmill exercise for 30 minutes once a day for 2 weeks. Step-down avoidance task and Y maze task were performed. Terminal deoxynucleotidyl transferase-mediated dUTP nick end labeling (TUNEL)staining, immunohistochemistry for 5-bromo-2'-deoxyridine, doublecortin, caspase-3, and Western blot for brain-derived neurotrophic factor (BDNF), Bax, Bcl-2, cytochrome $c$, caspase-3 were conducted.

Results: Ischemia caused memory impairment with an increase of cell proliferation, BDNF expression, and apoptosis in the hippocampus. Treadmill exercise improved memory function with further increase of cell proliferation and BDNF expression and a decrease of apoptosis.

Conclusions: The animal model that we have developed and our assessment of the relation between exercise and brain function can be useful tools for future investigations of NLUTD symptoms associated with stroke, particularly ischemic stroke. The present study suggests that treadmill exercise promoted the recovery of brain function after cerebral ischemia.

Keyword: Ischemia; Cell Proliferation; Brain-Derived Neurotrophic Factor; Apoptosis; Exercise Test

- Grant Support: This study was supported by grant from the Korea Institute of Sport Science (KISS-201308-IF-02).

- Research Ethics: All animal experimental procedures conformed to the regulations stipulated by the National Institutes of Health (NIH), and the guidelines of the Korean Academy of Medical Science. This study was approved by the Kyung Hee University Institutional Animal Care and Use Committee (Seoul, Korea) [KHUASP(SE)-14-018].

- Conflict of Interest: No potential conflict of interest relevant to this article was reported

\section{INTRODUCTION}

Neurogenic lower urinary tract dysfunction (NLUTD) symp- toms are a major problem in patients with a variety of neurological disorders, and may lead to debilitating symptoms and serious complications such as chronic renal failure and recurrent uri-

Corresponding author: Chang-Ju Kim (iD http://orcid.org/0000-0003-4749-5795 Department of Physiology, Kyung Hee University School of Medicine, 26 Kyungheedae-ro, Dongdaemun-gu, Seoul 130 701, Korea

E-mail: changju@khu.ac.kr / Tel: +82-2-961-0407 / Fax: +82-2-964-2195

Submitted: November 3, 2014 / Accepted after revision: December 9, 2014

(c) (1) (9) This is an Open Access article distributed under the terms of the Creative Commons Attribution Non-Commercial License (http://creativecommons.org/licenses/by-nc/3.0/) which permits unrestricted non-commercial use, distribution, and reproduction in any medium, provided the original work is properly cited. 
nary tract infections. Many animal studies of NLUTD have focused on animal models of cerebral ischemia [1]. Clinically, stroke is known to be associated with voiding dysfunction as well as several types of cognitive impairment. Cerebrovascular diseases are classified in two categories: ischemia, which is caused by a cerebrovascular blockage; and hemorrhage, which occurs due to a cerebrovascular rupture. Unfortunately, the incidence of cerebrovascular diseases is increasing as countries are faced with aging populations. Furthermore, stroke caused by cerebral ischemia is closely associated with the development of cognitive impairments [2].

The hippocampus is a region of the brain associated with cognitive functions, particularly with learning and memory. This brain region plays a critical role in the creation of new memories as well as in the processing of declarative and spatial memory [3]. Cerebral ischemia induces neuronal loss in the hippocampus, which leads to long-term memory deficits [4]. The hippocampus is a primary site of neurogenesis: a four-stage process where neuronal progenitors that originate from neural stem cells undergo proliferation, migration, survival, and differentiation into new neurons. For the detection of newly formed cells, 5bromo-2'-deoxyuridine (BrdU) and Ki-67 immunohistochemistry have been previously used [5-7]. A decrease in the number of cells labeled with these markers represents a reduction in cell proliferation, while an increase in the number of labeled cells correlates with new cell formation $[7,8]$. In animal models of cerebral ischemia, an increase in cell proliferation in the hippocampus has been repeatedly reported; and this phenomenon has been considered a compensatory and adaptive response in pathologic situations [9-11]. Doublecortin (DCX) is a brain-specific microtubule-associated protein that is involved in neuronal migration and differentiation, and is used as a marker of neuronal precursor cells $[12,13]$.

Neurogenesis is promoted by various neurotrophic factors, including brain-derived neurotrophic factor (BDNF). BDNF regulates the proliferation and differentiation of neuronal stem cells and progenitor cells at various sites in the brain [14]. Cerebral ischemia eventually induces neuronal cell death, but interestingly, it simultaneously initiates molecular and cellular repair mechanisms such as the activation of neurogenesis [15].

Apoptosis is a critical process for normal development and tissue homeostasis, however, its abnormal occurrence has been implicated in a number of disorders, including neurodegenerative diseases and stroke $[11,16,17]$. The terminal deoxynucleotidyl transferase-mediated dUTP nick-end labeling (TUNEL) is an assay that detects DNA fragmentation, which is characteristic of apoptotic cell death [16]. Caspase- 3 is the most widely studied member of the caspase family, and it is one of the key executors of apoptosis [11]. Activation of caspase-3 is an important hallmark of apoptosis following ischemic brain insults $[11,16]$. The cell-damaging mechanisms that are activated by ischemia are counteracted by mechanisms of cell survival, which include upregulation of the antiapoptotic molecules $\mathrm{Bcl}-2$ and $\mathrm{Bcl}-2 \mathrm{xL}$. The anti-apoptotic protein $\mathrm{Bcl}-2$ suppresses apoptosis, while the proapoptotic protein Bax promotes apoptosis [18].

It has been reported that exercise improves brain function after several forms of brain damage $[8,13,17]$. In the present study, we evaluated the effects of treadmill exercise on memory function, and its relation to cell proliferation and apoptosis, using a model of cerebral ischemia in gerbils.

\section{MATERIALS AND METHODS}

\section{Experimental Animals}

Mongolian gerbils weighing $30 \pm 2 \mathrm{~g}$ ( 8 weeks old) were used in this experiment. The gerbils were kept with controlled temperature $\left(20^{\circ} \mathrm{C} \pm 2^{\circ} \mathrm{C}\right)$ under a 12-hour light/dark cycle. The animals were allowed free access to food and water. All animal experimental procedures conformed to the regulations stipulated by the National Institutes of Health (NIH), and the guidelines of the Korean Academy of Medical Science. The animals were assigned randomly to four groups ( $\mathrm{n}=10$ per group): sham-operation group, sham-operation plus exercise group, ischemia-induction group, and ischemia-induction plus exercise group. All gerbils received an intraperitoneal injection of $50 \mathrm{mg} / \mathrm{kg} \mathrm{5-}$ bromo-2'-deoxyuridine (BrdU; Sigma Chemical Co., St. Louis, MO, USA) once a day for five consecutive days, 30 minutes before starting the treadmill exercises.

\section{Induction of Transient Global Ischemia}

To induce transient global ischemia in gerbils, the surgical procedures were performed following a previously described method $[10,11]$. The gerbils were anesthetized using $3 \%$ isoflurane in a 20\% $\mathrm{O}_{2}-77 \% \mathrm{~N}_{2}$ mixture. Both common carotid arteries (CCAs) were exposed by bilateral neck incision. The CCAs were occluded with aneurysm clips for 5 minutes, and the clips were then removed to restore cerebral blood flow. The rectal temperature was maintained at $37^{\circ} \mathrm{C} \pm 0.5^{\circ} \mathrm{C}$ with a heating lamp until the gerbil regained consciousness. After recovery, the animals were monitored for an additional 2 hours to prevent hypothermia. 
The gerbils in the sham-operation group and in the ischemiainduction group were treated identically, except that the CCAs were not occluded after the neck incision in the former group.

\section{Exercise Protocol}

The gerbils in the exercise groups were forced to run on a motorized treadmill for 30 minutes, once a day for 2 weeks. The exercise load consisted of running at a speed of $2 \mathrm{~m} / \mathrm{min}$ for the first 5 minutes, $5 \mathrm{~m} / \mathrm{min}$ for the following 5 minutes, and $8 \mathrm{~m} /$ min for the last 20 minutes, with zero degrees of inclination. The gerbils in the control groups were left on the treadmill without running for the same period as the exercise groups.

\section{Step-Down Avoidance Task}

To evaluate short-term memory we followed a previously described method [11] to determine the latency time in the stepdown avoidance task. The gerbils were trained in this task 14 days after starting the treadmill exercise. Two hours after training, the latency time (second) was measured for each group. The gerbils were placed on a $7 \times 25-\mathrm{cm}$ platform that was $2.5 \mathrm{~cm}$ in height. The platform faced a $42 \times 25-\mathrm{cm}$ grid of parallel stainless steel bars, $0.1 \mathrm{~cm}$ in caliber, spaced $1 \mathrm{~cm}$ apart. In the training sessions, the animals received a $0.5-\mathrm{mA}$ scramble foot shock for 2 seconds immediately after stepping down. The interval of time that elapsed between the gerbils stepping down and placing all four paws on the grid was defined as the latency time. A latency time $>180$ seconds was counted as 180 seconds.

\section{Y Maze Task}

Spontaneous alternation in the Y maze task was determined to evaluate spatial working memory, according to a previous study [19]. The gerbils were tested in the Y maze task 14 days after starting the treadmill exercise. The $\mathrm{Y}$ maze used in this study consisted of three arms (50 cm in length, $35 \mathrm{~cm}$ in height, and $12 \mathrm{~cm}$ width), and an equilateral triangular central area. For adaptation, the gerbils were allowed to move freely in the maze for 1 minute. Afterwards they were allowed to voluntarily enter the maze for 8 minutes. Spontaneous alternation was defined as consecutive entry into all three arms. The task was quantified as follows: percentage of spontaneous alternation $=$ (number of consecutive entrances into each arm/number of entrances into each arm2) $\times 100$.

\section{Tissue Preparation}

The gerbils were euthanized immediately after the determina- tion of latency in the step-down avoidance task. The gerbils were anesthetized using Zoletil 50 (10 mg/kg, intraperitoneally; Vibac Laboratories, Carros, France), and transcardially perfused with $50 \mathrm{mM}$ phosphate-buffered saline (PBS) followed by a freshly prepared solution of $4 \%$ paraformaldehyde in $100 \mathrm{mM}$ phosphate buffer ( $\mathrm{pH}$ 7.4). The brains were dissected, postfixed in the same fixative overnight, and transferred to a $30 \%$ sucrose solution for cryoprotection. Forty-micrometer-thick coronal sections were made using a freezing microtome (Leica, Nussloch, Germany). On average 10 sections through the hippocampus were collected from each gerbil.

\section{Immunohistochemistry for BrdU}

To detect newly generated cells in the hippocampal dentate gyrus, BrdU-specific immunohistochemistry was performed according to a previously described method [6,20]. The sections were first permeabilized by incubation in $0.5 \%$ Triton X-100 in PBS for 20 minutes, then pretreated in 50\% formamide- $2 \mathrm{X}$ standard saline citrate at $65^{\circ} \mathrm{C}$ for 2 hours, denatured in $2 \mathrm{~N} \mathrm{HCl}$ at $37^{\circ} \mathrm{C}$ for 30 minutes, and rinsed twice in $100 \mathrm{mM}$ sodium borate ( $\mathrm{pH}$ 8.5). Afterwards, the sections were incubated overnight at $4^{\circ} \mathrm{C}$ with a BrdU-specific mouse monoclonal antibody (1:600; Roche, Mannheim, Germany). The following day, the sections were washed three times with PBS, and incubated with a biotinylated antimouse secondary antibody (1:200; Vector Laboratories, Burlingame, CA, USA) for 1 hour. Then, the sections were incubated for 1 hour with an avidin-peroxidase complex (1:100; Vector Laboratories). For visualization, sections were incubated in $50 \mathrm{mM}$ Tris- $\mathrm{HCl}(\mathrm{pH} 7.6)$ containing $0.03 \%$ 3, 3'-diaminobenzidine (DAB), 40-mg/mL nickel chloride, and $0.03 \%$ hydrogen peroxide for 5 minutes.

After BrdU immunohistochemistry, the identification of Br$\mathrm{dU}$-positive neurons was done in the same section using a mouse anti-neuronal nucleic antibody (1:1,000; Chemicon International, Temecula, CA, USA). The sections were washed three times with PBS, and incubated with a biotinylated antimouse secondary antibody for 1 hour. For visualization, the sections were incubated in a reaction mixture consisting of $0.03 \% \mathrm{DAB}$ and $0.03 \%$ hydrogen peroxide for 5 minutes. The sections were mounted onto gelatin-coated slides, air-dried overnight at room temperature, and coverslipped using Permount (Fisher Scientific, New Jersey, NJ, USA).

\section{Immunohistochemistry for DCX and Caspase-3}

To visualize DCX and caspase-3 expression in the hippocampal 
dentate gyrus, immunohistochemistry was performed following a previously described method $[11,21]$. The sections were immersed 10 minutes in PBS and then washed three times in the same buffer. The sections were then incubated in a solution of $1 \%$ hydrogen peroxide in PBS for 30 minutes. Hippocampal sections from each animal were incubated overnight with a goat anti-DCX antibody (1:1,000; Oncogene Research Product, Cambridge, UK) and a rabbit anti-caspase-3 antibody (1:500, Abcam, Cambridge, UK). The following day, the sections were incubated with a biotinylated antirabbit secondary antibody (1: 200; Vector Laboratories) for 1 hour. The secondary antibody was amplified with the Vector Elite ABC kit (1:100; Vector Laboratories). The antibody-biotin-avidin-peroxidase complexes were visualized using $0.03 \% \mathrm{DAB}$, and the sections were mounted onto gelatin-coated slides. The slides were air-dried overnight at room temperature, and coverslipped using Permount (Fisher Scientific).

\section{TUNEL Staining}

To visualize DNA fragmentation, TUNEL staining was performed using an In Situ Cell Death Detection Kit (Roche), according to the manufacturer's protocol $[10,21]$. The sections were postfixed in ethanol-acetic acid (2:1 vol/vol) and rinsed. Afterwards, the sections were incubated with proteinase $\mathrm{K}(100 \mu \mathrm{g} / \mathrm{mL})$, rinsed, incubated in 3\% hydrogen peroxide, permeabilized with $0.5 \%$ Triton X-100, rinsed again, and incubated in the TUNEL reaction mixture. The sections were rinsed and visualized using Converter-POD with 0.03\% DAB. Mayer's hematoxylin (Dako, Glostrup, Denmark) was used as a counterstain, and sections were mounted onto gelatin-coated slides. The slides were airdried overnight at room temperature, and coverslipped using Permount (Fisher Scientific).

\section{Western Blot for BDNF, Bax, Bcl-2, Cytochrome c, and Caspase-3}

Western blots were performed according to a previously described protocol $[16,20]$. Hippocampal tissue samples were immediately frozen at $-70^{\circ} \mathrm{C}$ and stored at the same temperature until use. The hippocampal samples were homogenized using an ice-cold lysis buffer containing $50 \mathrm{mM}$ Tris- $\mathrm{HCl}$ ( $\mathrm{pH} 7.5$ ), $150 \mathrm{mM} \mathrm{NaCl}, 0.5 \%$ deoxycholic acid, 1\% Nonidet P40, 0.1\% sodium dodecyl sulfate (SDS), $1 \mathrm{mM}$ phenylmethylsulfonyl fluoride, and $100 \mathrm{mg} / \mathrm{mL}$ leupeptin. Total protein concentration was measured using a Bio-Rad colorimetric protein assay kit (Bio-Rad, Hercules, CA, USA). Thirty microgram of total pro- tein was separated on SDS-polyacrylamide gels and transferred onto a nitrocellulose membrane, which was consecutively incubated with a mouse $\beta$-actin antibody (1:1,000; Santa Cruz Biotechnology, Santa Cruz, CA, USA), rabbit BDNF and caspase-3 antibodies (1:1,000; Santa Cruz Biotechnology), and mouse Bax, Bcl-2, and cytochrome $c$ antibodies (1:1,000; Santa Cruz Biotechnology). Horseradish peroxidase-conjugated antirabbit and antimouse antibodies were used as secondary antibodies. The Western blot procedures were performed at room temperature except for the transfer of the proteins to the membrane.

\section{Data Analysis}

For the analysis of the expression of BDNF, Bax, Bcl-2, cytochrome $c$, and caspase- 3 , the detected bands were calculated densitometrically using Molecular Analyst ver 1.4.1 (Bio-Rad). The numbers of BrdU-positive, TUNEL-positive, and caspase-3-positive cells in the hippocampal dentate gyrus were counted hemilaterally under a light microscope (Olympus, Tokyo, Japan), and they were expressed as the number of cells per $\mathrm{mm}^{2}$ in the hippocampal dentate gyrus. The area of the hippocampal dentate gyrus was measured using Image-Pro Plus image analysis system (Media Cyberbetics Inc., Silver Spring, MD, USA). The data were analyzed with one-way analysis of variance followed by Duncan post hoc tests. All values are expressed as the mean \pm standard error of the mean, and a P-value of $<0.05$ was considered significant.

\section{RESULTS}

\section{Effects of Treadmill Exercise on Memory Function}

In order to investigate short-term memory, we used a step-down avoidance task. The results of the step-down avoidance task are presented in left panel of Fig. 1. The latency was $43.28 \pm 5.82 \mathrm{sec}-$ onds in the sham-operation group, $54.42 \pm 9.89$ seconds in the sham-operation and exercise group, $21.28 \pm 4.54$ seconds in the ischemia-induction group, and $43.14 \pm 6.98$ seconds in the ischemia-induction and exercise group. Latency was decreased by induction of ischemia, in contrast, treadmill exercise increased latency in the ischemic gerbils.

In order to investigate spatial working memory, we assessed spontaneous alternation in the Y maze task. The results of Ymaze task are presented in right panel of Fig. 1 . The $\%$ of spontaneous alternation was $69.00 \% \pm 2.65 \%$ in the sham-operation group, $70.16 \% \pm 2.38 \%$ in the sham-operation and exercise group, $40.50 \% \pm 3.75 \%$ in the ischemia-induction group, and $62.50 \% \pm 2.05 \%$ in the ischemia-induction and exercise group. 

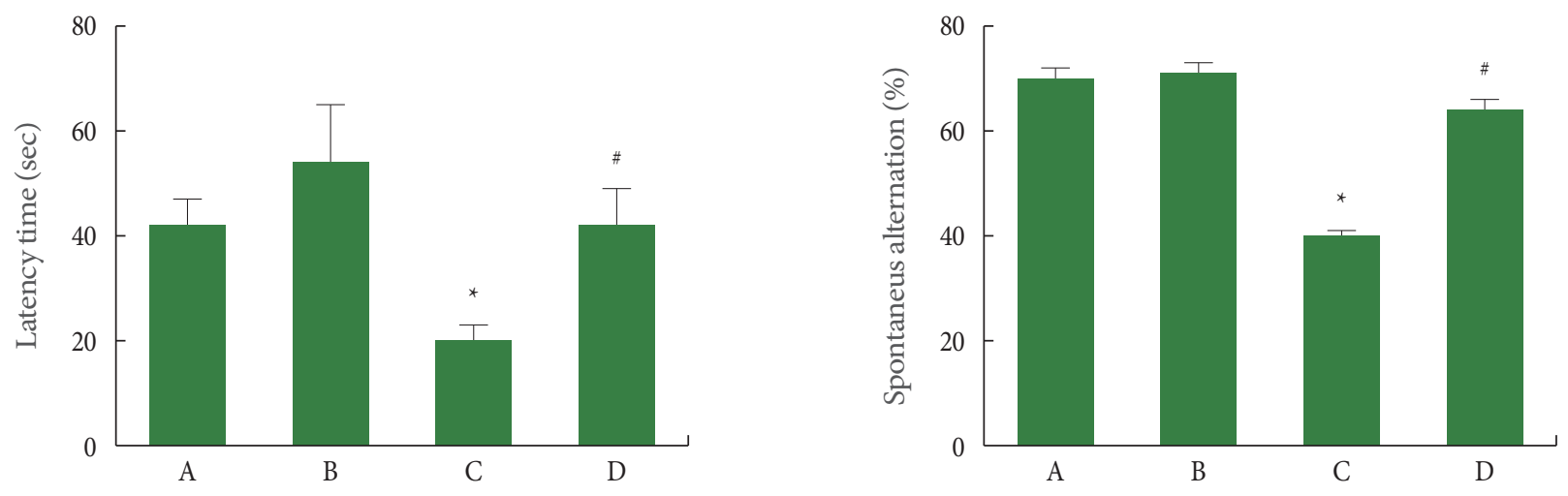

Fig. 1. Effect of treadmill exercise on memory function. Left: Step-down avoidance task, Right: Y-maze task. (A) Sham-operation group, (B) sham-operation plus exercise group, $(\mathrm{C})$ ischemia-induction group, and (D) ischemia-induction plus exercise group. ${ }^{*} \mathrm{P}<0.05$ compared to the sham-operation group. ${ }^{*} \mathrm{P}<0.05$ compared to the ischemia-induction group.
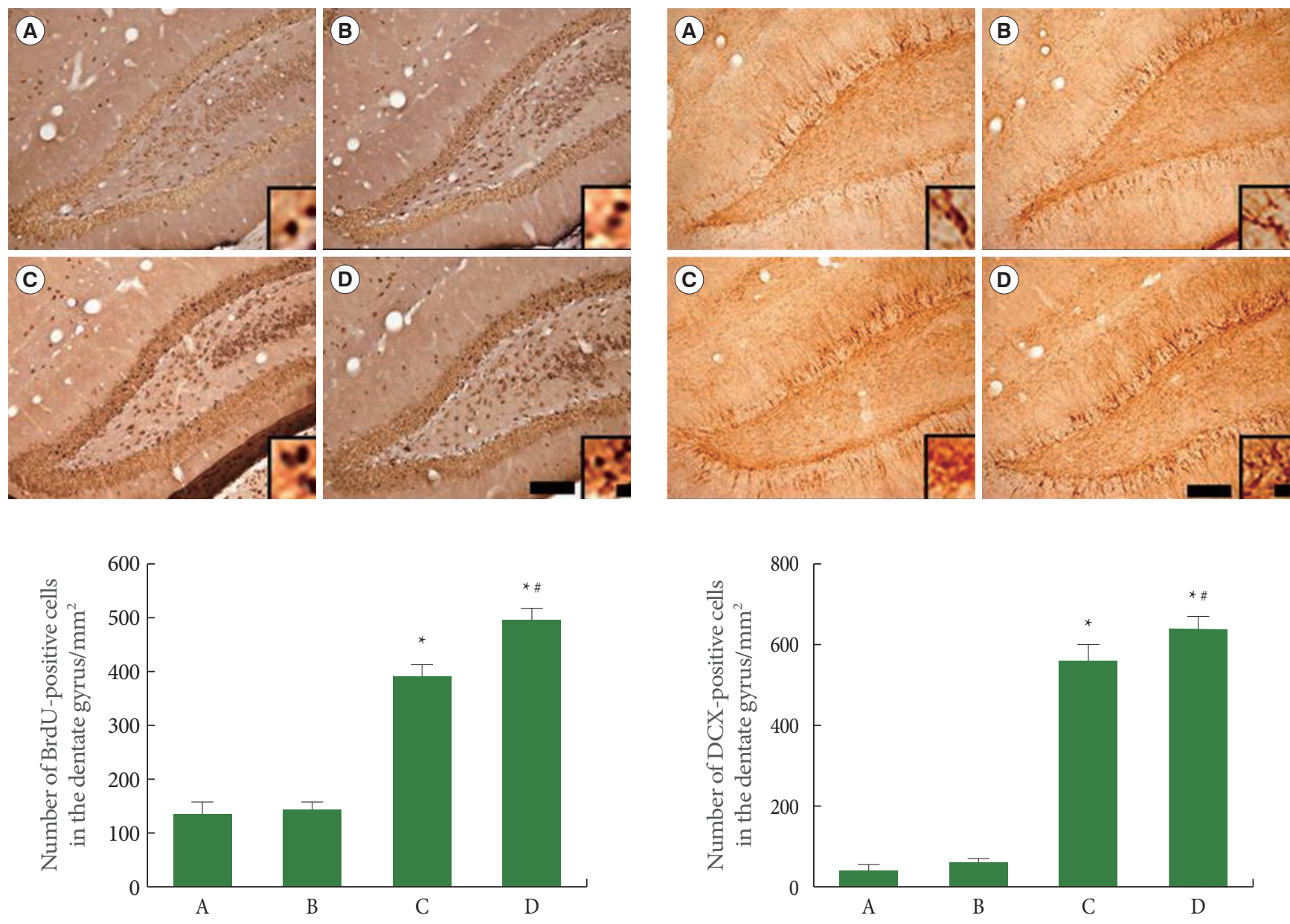

Fig. 2. Effect of treadmill exercise on cell proliferation in the hippocampal dentate gyrus. Left: 5-brmo-2'-deoxyuridine (BrdU) immunohistochemistry, Right: Doublecortin (DCX) immunohistochemistry. The scale bar represents $50 \mu \mathrm{m}$ (low magnification) and $10 \mu \mathrm{m}$ (high magnification, enclosed image). (A) Sham-operation group, (B) sham-operation plus exercise group, (C) ischemia-induction group, and (D) ischemia-induction plus exercise group. ${ }^{*} \mathrm{P}<0.05$ compared to the sham-operation group. ${ }^{*} \mathrm{P}<0.05$ compared to the ischemia-induction group. 


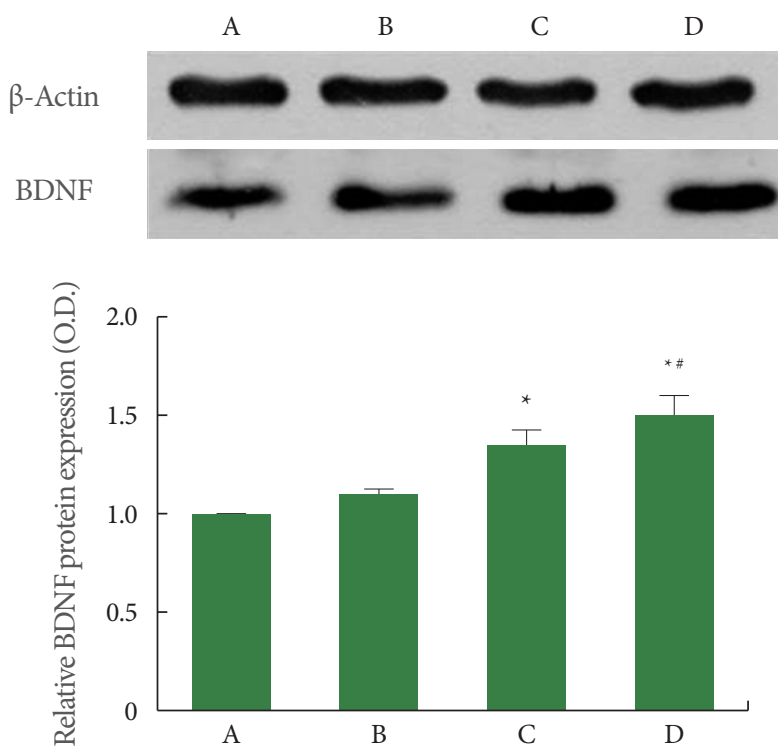

Fig. 3. Effect of treadmill exercise on brain-derived neurotrophic factor (BDNF) expression in the hippocampus. (A) Sham-operation group, (B) sham-operation plus exercise group, (C) ischemiainduction group, and (D) ischemia-induction plus exercise group. ${ }^{*} \mathrm{P}<0.05$ compared to the sham-operation group. ${ }^{*} \mathrm{P}<0.05$ compared to the ischemia-induction group.

Spontaneous alternation was decreased by induction of ischemia, in contrast, treadmill exercise increased spontaneous alternation in the ischemic gerbils.

\section{Effects of Treadmill Exercise on Cell Proliferation in Hippocampal Dentate Gyrus}

We used BrdU and DCX immunohistochemistry to assess cell proliferation in the hippocampal dentate gyrus. The results of $\mathrm{Br}$ dU-positive and DCX-positive cells in the hippocampal dentate gyrus are presented in Fig. 2. The number of BrdU-positive cells was $146.44 \pm 13.66$ in the sham-operation group, $150.90 \pm 11.80$ in the sham-operation plus exercise group, $388.75 \pm 29.79$ in the ischemia-induction group, and $484.09 \pm 25.70$ in the ischemiainduction plus exercise group. The number of BrdU-positive cells was increased by the induction of ischemia. In addition, treadmill exercise further increased the number of BrdU-positive cells in the ischemic gerbils.

The number of DCX-positive cells was $54.90 \pm 8.18$ in the sham-operation group, $64.75 \pm 14.15$ in the sham-operation plus exercise group, $540.88 \pm 47.25$ in the ischemia-induction group, and $643.61 \pm 38.68$ in the ischemia-induction plus exercise group. The number of DCX-positive cells was increased by the induction of ischemia. In addition, treadmill exercise further increased the number of DCX-positive cells in the ischemic gerbils.

\section{Effects of Treadmill Exercise on BDNF Expression in Hippocampus}

The expression of the mature form of BDNF $(14 \mathrm{kDa})$ was quantified using Western blot. The results of BDNF expression in the hippocampus are presented in Fig. 3. When the level of BDNF expression in the sham-operation group was set at 1.00 (baseline), the level of BDNF expression was $1.14 \pm 0.01$ in the sham-operation plus exercise group, $1.48 \pm 0.09$ in the ischemiainduction group, and $1.65 \pm 0.12$ in the ischemia-induction plus exercise group. BDNF expression was increased by the induction of ischemia, while treadmill exercise further increased $\mathrm{BDNF}$ expression in the ischemic gerbils.

\section{Effects of Treadmill Exercise on Apoptotic Neuronal Cell Death in Hippocampal Dentate Gyrus}

Apoptotic neuronal death was evaluated using TUNEL staining and caspase-3 immunohistochemistry (Fig. 4). The number of TUNEL-positive cells was $14.29 \pm 3.12$ in the sham-operation group, $18.58 \pm 3.81$ in the sham-operation plus exercise group, $43.34 \pm 6.16$ in the ischemia-induction group, and $29.36 \pm 5.18$ in the ischemia-induction plus exercise group. The number of TUNEL-positive cells was increased by the induction of ischemia, while treadmill exercise decreased the number of TUNELpositive cells in the ischemic gerbils.

The number of caspase-3-positive cells was $106.43 \pm 12.79$ in the sham-operation group, $110.95 \pm 12.27$ in the sham-operation plus exercise group, $252.95 \pm 38.74$ in the ischemia-induction group, and $180.30 \pm 16.10$ in the ischemia-induction plus exercise group. The number of caspase-3-positive cells was increased by the induction of ischemia, while the treadmill exercise decreased the number of caspase-3-positive cells in the ischemic gerbils.

\section{Effects of Treadmill Exercise on Bax and Bcl-2 Expression in Hippocampus}

The expression of the monomer forms of $\mathrm{Bax}(23 \mathrm{kDa})$ and $\mathrm{Bcl}-$ $2(26 \mathrm{kDa})$ was quantified using western blot, and the results are presented in Fig. 5. When the level of Bax expression in the sham-operation group was set at 1.00 (baseline), the level of Bax expression was $0.83 \pm 0.02$ in the sham-operation plus exercise group, $1.39 \pm 0.24$ in the ischemia-induction group, and $0.82 \pm 0.03$ in the ischemia-induction plus exercise group. The expression of Bax was increased by the induction of ischemia, 

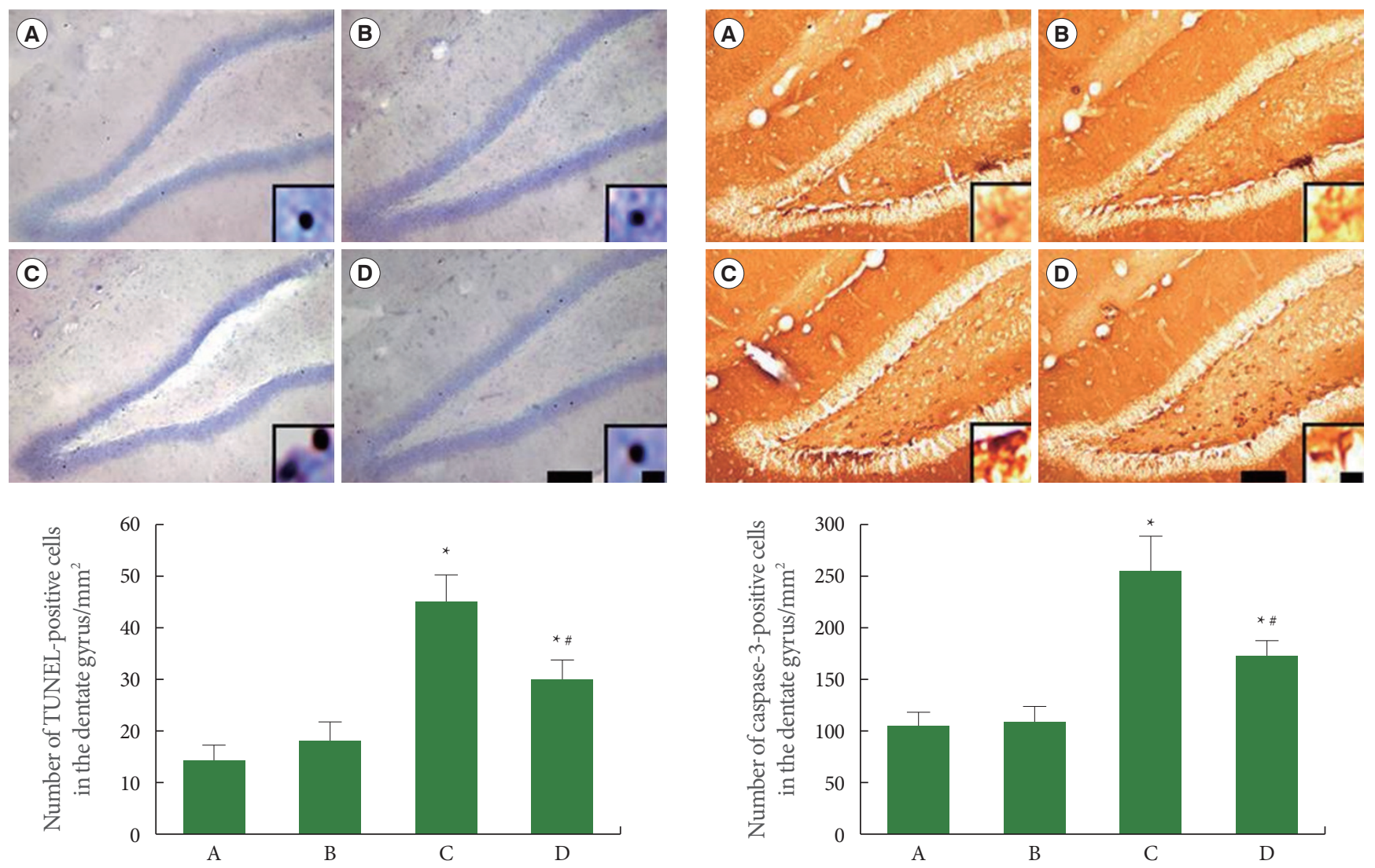

Fig. 4. Effect of treadmill exercise on the number of terminal deoxynucleotidyl transferase-mediated dUTP nick end-labeling (TUNEL)positive and caspase-3-positive cells in the hippocampal dentate gyrus. Left: TUNEL staining, Right: Caspase-3 immunohistochemistry. The scale bar represents $50 \mu \mathrm{m}$ (low magnification) and $10 \mu \mathrm{m}$ (high magnification, enclosed image). (A) Sham-operation group, (B) sham-operation plus exercise group, $(\mathrm{C})$ ischemia-induction group, and (D) ischemia-induction plus exercise group. ${ }^{*} \mathrm{P}<0.05$ compared to the sham-operation group. ${ }^{"} \mathrm{P}<0.05$ compared to the ischemia-induction group.

while treadmill exercise decreased the expression of Bax in the ischemic gerbils.

When the level of Bcl-2 expression in the sham-operation group was set at 1.00 (baseline), the level of Bcl-2 expression was $0.94 \pm 0.02$ in the sham-operation plus exercise group, $0.49 \pm 0.03$ in the ischemia-induction group, and $0.99 \pm 0.04$ in the ischemia-induction plus exercise group. The expression of $\mathrm{Bcl}-2$ was inhibited by the induction of ischemia, while treadmill exercise increased the expression of $\mathrm{Bcl}-2$ in the ischemic gerbils.

When the ratio of Bax to $\mathrm{Bcl}-2$ in the sham-operation group was set at 1.00 (baseline), the ratio of Bax to Bcl- 2 was $0.89 \pm 0.04$ in the sham-operation plus exercise group, $3.03 \pm 0.38$ in the ischemia-induction group, and $0.83 \pm 0.03$ in the ischemia-induction plus exercise group. The ratio of Bax to $\mathrm{Bcl}-2$ was increased by the induction of ischemia, while treadmill exercise decreased the ratio of $\mathrm{Bax}$ to $\mathrm{Bcl}-2$ in the ischemic gerbils.

\section{Effects of Treadmill Exercise on Cytochrome $c$ and Active Caspase-3 Expression in Hippocampus}

The expression of cytochrome $c(15 \mathrm{kDa})$ and caspase-3 $(17$ $\mathrm{kDa}$ ) was quantified using western blot, and the results are presented in Fig. 6. When the level of cytochrome $c$ expression in the sham-operation group was set at 1.00 (baseline), the level of cytochrome $c$ expression was $0.63 \pm 0.08$ in the sham-operation plus exercise group, $1.52 \pm 0.03$ in the ischemia-induction group, and $1.21 \pm 0.06$ in the ischemia-induction plus exercise group. Cytochrome $c$ expression was increased by the induction of ischemia, while treadmill exercise decreased the expression of cytochrome $c$ in the ischemic gerbils.

When the level of caspase- 3 expression in the sham-operation group was set at 1.00 (baseline), the level of caspase-3 expression was $0.99 \pm 0.01$ in the sham-operation plus exercise group, $2.13 \pm 0.07$ in the ischemia-induction group, and $1.78 \pm 0.06$ in 

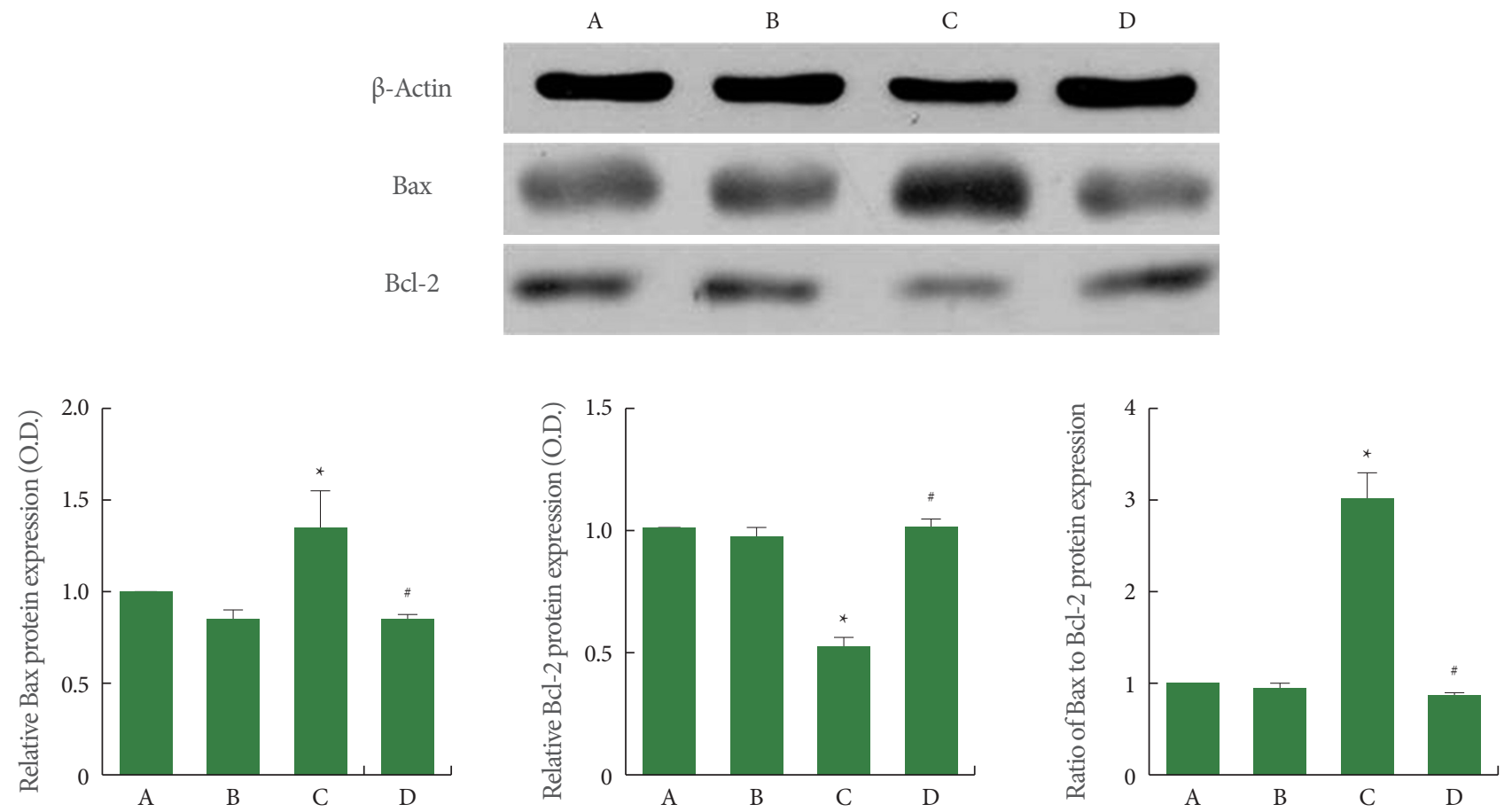

Fig. 5. Effect of treadmill exercise on Bax and Bcl-2 expression in the hippocampus. (A) Sham-operation group, (B) sham-operation plus exercise group, $(\mathrm{C})$ ischemia-induction group, and (D) ischemia-induction plus exercise group. ${ }^{*} \mathrm{P}<0.05$ compared to the sham-operation group. ${ }^{*} \mathrm{P}<0.05$ compared to the ischemia-induction group.
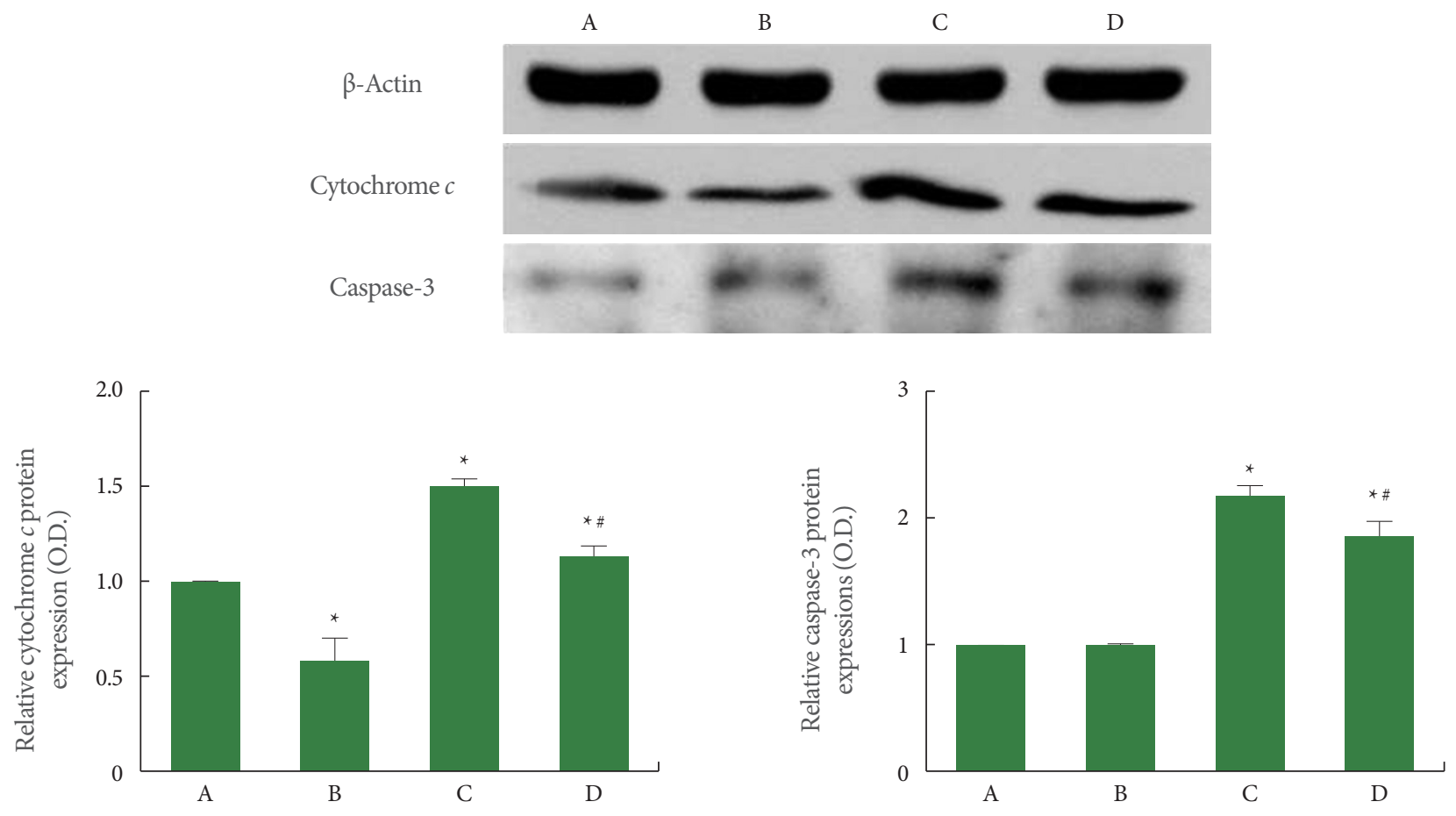

Fig. 6. Effect of treadmill exercise on cytochrome $c$ and caspase-3 expression in the hippocampus. (A) Sham-operation group, (B) shamoperation plus exercise group, (C) ischemia-induction group, and (D) ischemia-induction plus exercise group. ${ }^{\star} \mathrm{P}<0.05$ compared to the sham-operation group. ${ }^{\#} \mathrm{P}<0.05$ compared to the ischemia-induction group. 
the ischemia-induction plus exercise group. The expression of caspase- 3 expression was increased by the induction of ischemia, while treadmill exercise decreased caspase-3 expression in the ischemic gerbils.

\section{DISCUSSION}

Cerebral damage induced by ischemia negatively affects intelligence, memory, executive function, and language skills $[2,10,11]$. In the present study, short-term memory and spatial working memory deteriorated as a consequence of cerebral ischemia. Memory impairment is closely associated with hippocampal neurogenesis, BDNF, and apoptosis [11,17,22].

Pathological conditions such as cerebral ischemia increase hippocampal neurogenesis $[9,10,23]$. Liu et al. [9] reported that cell proliferation in the hippocampal dentate gyrus of gerbils was increased significantly after ischemia, reaching a maximum level at eleven days after the insult. Since Liu's initial study using gerbils, other reports have consistently shown that induction of ischemia in adult rodents produced an increase in the rate of cell proliferation in the hippocampal dentate gyrus and in the cerebral cortex $[10,23]$. An enhanced cell proliferation in the hippocampal dentate gyrus is known as a compensatory and adaptive response to excessive apoptosis [9-11]. Meanwhile, BDNF may play a protective role in the mechanism of neuronal cell death after cerebral ischemia [24], and BDNF expression was increased in the four-vessel occlusion rat model of transient global ischemia [25]. In addition, it has been suggested that an increase in BDNF expression may be a compensatory response to cerebral ischemia $[24,25]$. In the present study, the number of BrdUand DCX-positive cells in the hippocampal dentate gyrus increased, and the level of BDNF expression in the hippocampus was also increased by the induction of cerebral ischemia.

During cerebral ischemia, complex pathophysiological processes result in irreversible neurological damage [26]. After focal cerebral ischemia, neurons at the ischemic site showed DNA fragmentation, a common sign of apoptotic cell death [10,11]. It has been shown that cerebral ischemia leads to an increment in caspase- 3 levels, while the suppression of caspase- 3 expression reduced neuronal cell death induced by ischemia $[10,11]$. In the present study, the number of TUNEL- and caspase-3-positive cells in the hippocampal dentate gyrus increased following cerebral ischemia.

Bax and Bcl-2 are two distinct members of a family of proteins that regulate apoptosis, and their individual products possess op- posing functions. Bcl-2 is functionally characterized as the apoptosis-suppressing factor, whereas Bax is considered as the apoptosis-promoting factor [16]. Bcl-2 inhibits apoptosis by preventing the release of cytochrome $c$ from the mitochondria, and Bax is the dominant proapoptotic protein involved in the release of $\mathrm{cy}$ tochrome $c$ [18]. In the present study, cerebral ischemia increased the expression of cytochrome $c$ and active caspase- 3 in the hippocampus. Bcl-2 expression in the hippocampus was decreased, while Bax expression was increased after cerebral ischemia.

The memory-improving effect of exercise is well documented $[8,27,28]$. Short-term memory impairments induced by cerebral ischemia were improved by treadmill exercise [10]. Improvement in short-term memory was achieved by activation of neurogenesis, increase of BDNF expression, and inhibition of apoptosis in the hippocampus $[8,10,11]$. In the present study, impairment of short-term memory and spatial working memory caused by cerebral ischemia was alleviated by treadmill exercise.

Lee et al. [29] demonstrated that the number of BrdU- and DCX-positive cells in the hippocampal dentate gyrus increased following ischemia, while physical training further increased those numbers. In addition, the use of an enriched environment, such as running wheels and tunnels in the cages, further increased BDNF expression in ischemic rats, resulting in an improvement of their learning ability and memory capability [25]. Such results might be considered as a synergistic effect of exercise. In the present study, the number of BrdU- and DCX-positive cells and BDNF expression increased following cerebral ischemia, and this increase was potentiated by treadmill exercise.

The antiapoptotic effect of treadmill exercise on brain insults is well documented $[10,17,21]$. Treadmill exercise for 4 weeks inhibited the ischemia-induced increase in the number of TUNEL- and caspase-3-positive cells in the hippocampus of gerbils [10]. In the present study, treadmill exercise decreased the number of TUNEL- and caspase-3-positive cells in the hippocampal dentate gyrus of ischemic gerbils. Treadmill exercise also resulted in a decrease of Bax to Bcl-2 ratio through an increase in Bcl2 expression, and a decrease in Bax expression. A reduction in cytochrome $c$ and active caspase-3 expression was also observed following treadmill exercise in ischemic gerbils.

There is little consensus about how stroke patients with NLUTD symptoms should be managed, and current rehabilitation options for NLUTD are also limited. The animal model that we have developed and our assessment of the relation between exercise and brain function can be useful tools for future investigations of NLUTD symptoms associated with stroke, particularly 
ischemic stroke. The enhancement of neurogenesis and the inhibition of apoptosis produced by treadmill exercise may help to improve memory function. The present study suggests that treadmill exercise promoted the recovery of brain function after cerebral ischemia.

\section{REFERENCES}

1. Fry CH, Daneshgari F, Thor K, Drake M, Eccles R, Kanai AJ, et al. Animal models and their use in understanding lower urinary tract dysfunction. Neurourol Urodyn 2010;29:603-8.

2. Mori E. Impact of subcortical ischemic lesions on behavior and cognition. Ann N Y Acad Sci 2002;977:141-8.

3. Biegler R, McGregor A, Krebs JR, Healy SD. A larger hippocampus is associated with longer-lasting spatial memory. Proc Natl Acad U S A 2001;98:6941-4.

4. Hartman RE, Lee JM, Zipfel GJ, Wozniak DF. Characterizing learning deficits and hippocampal neuron loss following transient global cerebral ischemia in rats. Brain Res 2005;1043:48-56.

5. Kim H, Lee MH, Chang HK, Lee TH, Lee HH, Shin MC, et al. Influence of prenatal noise and music on the spatial memory and neurogenesis in the hippocampus of developing rats. Brain Dev 2006; 28:109-14.

6. Lee SW, Kim YS, Jun TW, Seo JH, Kim K, Shin MS, et al. The impact of duration of one bout treadmill exercise on cell proliferation and central fatigue in rats. J Exerc Rehabil 2013;9:463-9.

7. Sung YH, Shin MS, Cho S, Baik HH, Jin BK, Chang HK, et al. Depression-like state in maternal rats induced by repeated separation of pups is accompanied by a decrease of cell proliferation and an increase of apoptosis in the hippocampus. Neurosci Lett 2010;470: $86-90$.

8. Kim TW, Shin MS, Park JK, Shin MA, Lee HH, Lee SJ. Treadmill exercise alleviates prenatal noise stress-induced impairment of spatial learning ability through enhancing hippocampal neurogenesis in rat pups. J Exerc Rehabil 2013;9:451-6.

9. Liu J, Solway K, Messing RO, Sharp FR. Increased neurogenesis in the dentate gyrus after transient global ischemia in gerbils. J. Neurosci 1998;18:7768-78.

10. Sim YJ, Kim H, Kim JY, Yoon SJ, Kim SS, Chang HK, et al. Longterm treadmill exercise overcomes ischemia-induced apoptotic neuronal cell death in gerbils. Physiol Behav 2005;84:733-8.

11. Ko IG, Shin MS, Kim BK, Kim SE, Sung YH, Kim TS, et al. Tadalafil improves short-term memory by suppressing ischemia-induced apoptosis of hippocampal neuronal cells in gerbils. Pharmacol Biochem Behav 2009;91:629-35.
12. Friocourt G, Liu JS, Antypa M, Rakic S, Walsh CA, Parnavelas JG. Both doublecortin and doublecortin-like kinase play a role in cortical interneuron migration. J Neurosci 2007;27:3875-83.

13. Kim BK, Seo JH. Treadmill exercise alleviates post-traumatic stress disorder-induced impairment of spatial learning memory in rats. J Exerc Rehabil 2013;9:413-9.

14. Barnabe-Heider F, Miller FD. Endogenously produced neurotrophins regulate survival and differentiation of cortical progenitors via distinct signaling pathways. J Neurosci 2003;23:5149-60.

15. Cramer SC, Chopp M. Recovery recapitulates ontogeny. Trends Neurosci 2000;23:265-71.

16. Hwang L, Choi IY, Kim SE, Ko IG, Shin MS, Kim CJ, et al. Dexmedetomidine ameliorates intracerebral hemorrhage-induced memory impairment by inhibiting apoptosis and enhancing brain-derived neurotrophic factor expression in the rat hippocampus. Int J Mol Med 2013;31:1047-56.

17. Kim DH, Ko IG, Kim BK, Kim TW, Kim SE, Shin MS, et al. Treadmill exercise inhibits traumatic brain injury-induced hippocampal apoptosis. Physiol Behav 2010;101:660-5.

18. Jemmerson R, Dubinsky JM, Brustovetsky N. Cytochrome c release from CNS mitochondria and potential for clinical intervention in apoptosis-mediated CNS diseases. Antioxid Redox Signal 2005;7: 1158-72.

19. Bild W, Hritcu L, Stefanescu C, Ciobica A. Inhibition of central angiotensin II enhances memory function and reduces oxidative stress status in rat hippocampus. Prog Neuropsychopharmacol Biol Psychiatry 2013;3;43:79-88.

20. Kim JE, Ji ES, Seo JH, Lee MH, Cho S, Park YK, et al. Alcohol exposure induces depression-like behavior by decreasing hippocampal neuronal proliferation through inhibition of the BDNF-ERK pathway in gerbils. Anim Cells Syst 2012;16:190-7.

21. Ji ES, Ko IG, Cho JW, Davis RW, Hwang GY, Jee YS, et al. Treadmill exercise inhibits apoptotic neuronal cell death with suppressed vascular endothelial growth factor expression in the retinas of the diabetic rats. J Exerc Rehabil 2013;9:348-53.

22. Blurton-Jones M, Kitazawa M, Martinez-Coria H, Castello NA, Müller FJ, Loring JF, et al. Neural stem cells improve cognition via BDNF in a transgenic model of Alzheimer disease. Proc Natl Acad Sci U S A 2009;106:13594-9.

23. Yagita Y, Kitagawa K, Ohtsuki T, Takasawa Ki, Miyata T, Okano H, et al. Neurogenesis by progenitor cells in the ischemic adult rat hippocampus. Stroke 2001;32:1890-6.

24. Tsukahara T, Iihara K, Hashimoto N, Nishijima T, Taniguchi T. Increases in levels of brain-derived neurotrophic factor mRNA and its promoters after transient forebrain ischemia in the rat brain. 
Neurochem Int 1998;33:201-7.

25. Gobbo OL, O'Mara SM. Impact of enriched-environment housing on brain-derived neurotrophic factor and on cognitive performance after a transient global ischemia. Behav Brain Res 2004;152:231-41.

26. Broughton BR1, Reutens DC, Sobey CG. Apoptotic mechanisms after cerebral ischemia. Stroke 2009;40:e331-9.

27. Vaynman S, Ying Z, Gomez-Pinilla F. Hippocampal BDNF mediates the efficacy of exercise on synaptic plasticity and cognition.
Eur J Neurosci 2004;20:2580-90.

28. Uysal N, Tugyan K, Kayatekin BM, Acikgoz O, Bagriyanik HA, Gonenc $S$, et al. The effects of regular aerobic exercise in adolescent period on hippocampal neuron density, apoptosis and spatial memory. Neurosci Lett 2005;383:241-5.

29. Lee SH, Kim YH, Kim YJ, Yoon BW. Enforced physical training promotes neurogenesis in the subgranular zone after focal cerebral ischemia. J Neurol Sci 2008;269:54-61. 